\title{
USB Based Battery Independent Device for Pulse Measurement
}

\author{
Pranmya Ratnaparkhi \\ Student, VIT University, Bhopal, India
}

\begin{abstract}
Pulse measurement is considered one of the important parameters for diagnosis of various abnormalities. There are many devices available in the market which is used for pulse measurement but they are quite expensive, bulky and complex to use. So for overcoming these problems we tried to design a device which is independent of battery and a display which makes it highly compact and also works fine with almost all devices which supports OTG. No dedicated app is required for operating the device and can work with all kinds of messaging apps. As soon as it will detect the pulse a proper report containing the measured values will appear in the specific messaging app. Also the device is compatible with all kinds of the computers and laptops. The device works on the principle of photoplethysmography as it measures the pulse rate. The microcontroller processes the data and sends them to the device to which it is connected. It is highly useful for the people who need to measure their pulse rate very frequently like heart patients, Athletes etc.
\end{abstract}

Keywords: Pulse Rate, OTG, photoplethysmography, microcontroller.

\section{INTRODUCTION}

The system designed here is used to measure the pulse rate in order to diagnose for any kind of heart related abnormalities like blockage which may result in abnormal beating of heart. There may be many factors behind these kinds of abnormalities in the heart because of which there may be deviation in the heart beat. If the heartbeat goes down below 60 BPM then this condition is known as bradycardia and if the heartbeat is above 120 BPM then this condition is known as tachycardia. Both conditions are not good for the health of a person. For identifying and eliminating this kind of abnormalities the device designed is a good option as it is quite simple to operate and very compact as it is very small. The device does not use any kind of battery; it takes power from the device with which it is connected. Also it does not use any kind of display as it just displays the value on the device like mobile, $\mathrm{PC}$ etc with which it is connected, which reduces the power consumption and reduces the size of the device. It does not require any specific app for its operation; it can be operated with any app like notepad, any kind of messaging app etc. The device records all the values on the device in the form of text which is very easy to save, reduces size of the file and can be easily transferred to anyone.

The device is based upon the pulse sensor which works on the principle of photoplethysmography. This device is highly compact and is highly useful for the peoples who need to measure their pulse rate very frequently like the athletes for which pulse rate is one of the important parameter so this device can be used as it is very compact, does not require any kind of battery and also works fine with all kinds of devices.

\section{MATERIALS AND METHODS}

\subsection{Arduino}

Arduino is an open source development board which is dependent upon various types of microcontrollers like atmega328, $32 \mathrm{u} 4$ etc. It provides an open platform for development of the projects. It also uses some 32 bit microcontrollers like some based on ARM. Arduino is board equipped with many features. It has both analog and digital input and outputs. It has 6 analog pins and 14 digital pins of which some pins also act as PWM pins. It works on $5 \mathrm{v}$ and can take input voltage upto $12 \mathrm{v}$. This board has many types of peripheral interfaces. It allows transfer of data through I2C, SPI, UART protocols. It uses an oscillator of $16 \mathrm{MHz}$ along with on board USB to TTL converter chip. Arduino boards use a preloaded boot loader for easy uploading of code to the microcontroller flash memory.

There are many variants of Arduino boards depending upon the size and different processing units, like Arduino Uno, Mega, Nano, Pro Micro, Lonardo, Lillypad, and Due etc. All this board differs in either size or processing units. The board used in this project is Arduino Pro Micro which uses an Atmega32u4 microcontroller chip. Purpose of using this specific board is that it includes all the features of the Arduino board and also has an additional feature that it can be used as an USB device, which makes it very useful for this device. Just like Arduino uno Pro Micro also possesses all peripherals like I2C, SPI, UART etc and analog and digital pins. 


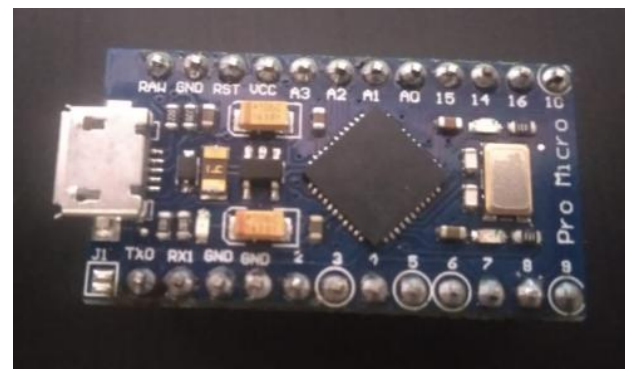

\subsection{Pulse Sensor}

Pulse sensor is a well designed sensor compatible with arduino and many other development boards. It is used for measurement of the pulse rate. It takes the measurement from fingertip or from the ear lobe. It has 3 pins i.e VCC, GND and signal pin. Out of these pins output signal is taken from the signal pin. The front side of the sensor which connects with skin has a heart logo and an opening for the light emitted by the led to pass through. Just below the hole there is a square in the ambient light sensor which detects the light. The LED shines the light onto the fingertip or the earlobe and the reflected light is analyzed by the ambient light sensor. The data from the sensor is recorded, processed by the microcontroller and then the suitable output is given.

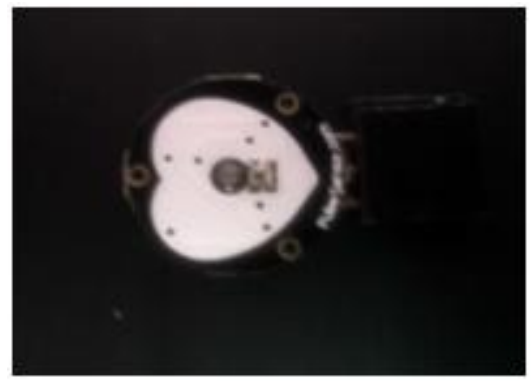

\subsection{USB Cable and OTG}

USB cable and OTG are required for connecting the device with host devices like mobile and PC's. OTG is required specifically for the mobile phones whereas the device can be directly connected to the PC's via USB cable. USB cable has two major functions; one is to supply power to the device from the USB port and second is to transfer the data between two devices. OTG pins can be used according to the mobile with which the device is used but for USB, B type cable should be used to connect the device.

\subsection{Arduino IDE}

Arduino IDE is an open source development environment for writing and deploying the code to different arduino and various other boards. It provides wide support for various other boards also. It has very large collections of many libraries and also supports many types of programmers required for different boards. Also has an inbuilt compiler
Volume 5, Issue 4, pp 83-86, April-2021

https://doi.org/10.47001/IRJIET/2021.504013

which helps in identifying the problem. This whole setup is written in JAVA.

\subsection{Methods}

The method used for the measurement of the pulse rate is photo-plethysmography. The basic principle behind the operation in this method is to measure pulsatile blood volume changes by optical methods. The device sends the light through the tissue and then the light is absorbed by the tissues. The LED emits the light which travels through the tissue. The photo resistor mounted on the device measures change in light intensity. With each contraction of the heart the volume of the blood is forced to their extreme value which alters the amount of the blood in the given volume which results in the change of optical density. This change in optical density per seconds is measured and the pulse rate is analyzed. The signal obtained from the phototransistor is amplified and filtered and then the time interval between two successive pulses is measured. Basically the circuit consists of two parts one is LED and the other one is driver circuit which is responsible for the amplification and denoising of the signals obtained from the photosensitive element. This signal obtained at the output of the pulse sensor is transferred to the microcontroller unit which reads the data and then performs some processing tasks for analyzing the signals and generate output accordingly. There are many different algorithms used for the calculation of the pulse rate from the raw data obtained by the pulse sensor. These algorithms are programmed into the microcontroller in order to get the desired output. The microcontrollers like arduino make use of their interrupts in order to properly process the data. In our device the sensor is interfaced with the Arduino Pro Micro.

Sometimes it becomes more useful and dependable to measure the peripheral pulse rate rather than getting the value derived from the regular ECG in case of the blockage as any abnormalities in it indicates the cessation of the blood through the vessels more specifically in the limb terminals. And also photoelectric measurement is much easier than taking the ECG.

\section{WORKING}

The device designed is able to measure the peripheral pulse rate i.e it is able to measure the pulse rate from the fingertip or the earlobe. The pulse sensor used in the device measures the pulse rate and then sends the signal to the arduino pro micro which processes the data and then displays the output in the text format on the device connected. The algorithms are programmed using some specific libraries which help to process the raw data. The basic working of the device is based upon the feature of the Arduino Pro Micro which allows it to act as an USB device. The Arduino Pro 
ISSN (online): 2581-3048

Micro makes use of various inbuilt libraries which allows it to read the data from the pulse sensor. Also some specific libraries are used for the Arduino Pro Micro which allows it to act as a USB device.

The basic principle behind the working of the device is that the Arduino Pro Micro reads the data from the pulse sensor and then acts as an USB device which generates the output in text format on the host device with which our device is connected. The power required to drive the circuit is taken from the host device via USB cable which makes the device battery independent as it does not use any kind of batteries for the power. The device does not use any kind of inbuilt display to display the output data instead of this the device itself acts as an USB device and displays the data onto the host device in the text format.

This further reduces the size of the device as it does use any kind of inbuilt display. The device works fine with the smartphones and PC and also works fine with apps like whatsapp, email, notepad etc. The data recorded in the text format can be easily sent to anyone via different apps. It becomes very easy to store the data in the form of text. The device is highly compact as no external batteries and internal displays are used.

\section{RESULTS}

The device works with both mobile and PC and also works with various messaging and text editing apps.

\subsection{Pulse measurement when connected with mobile}

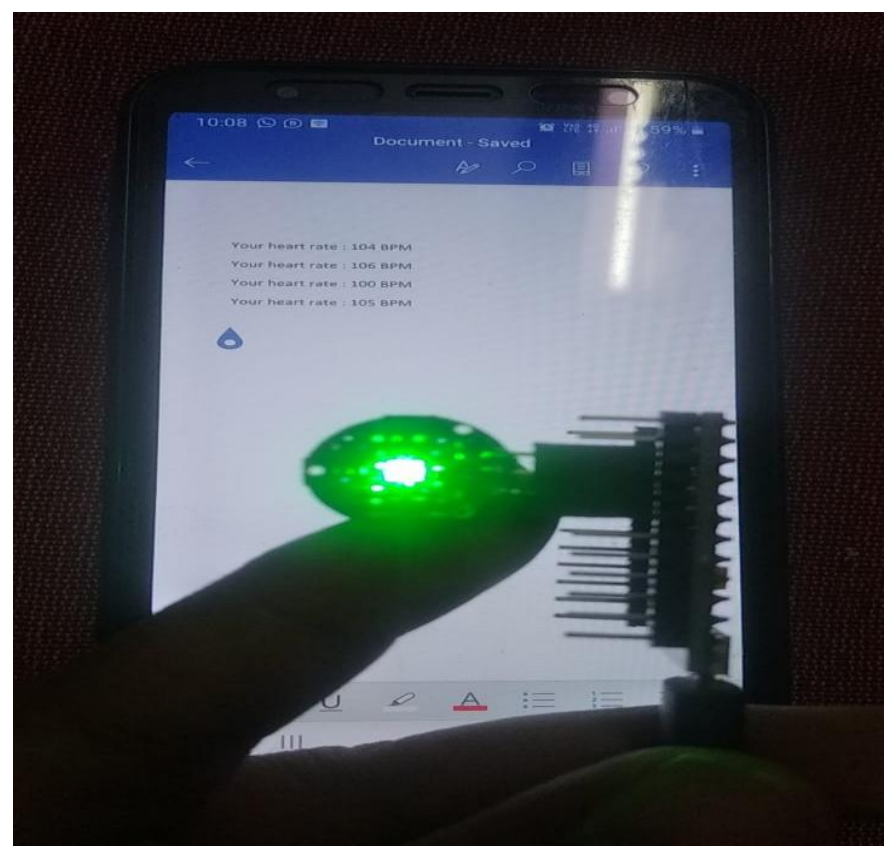

4.2 Pulse measurement when connected with PC on notepad

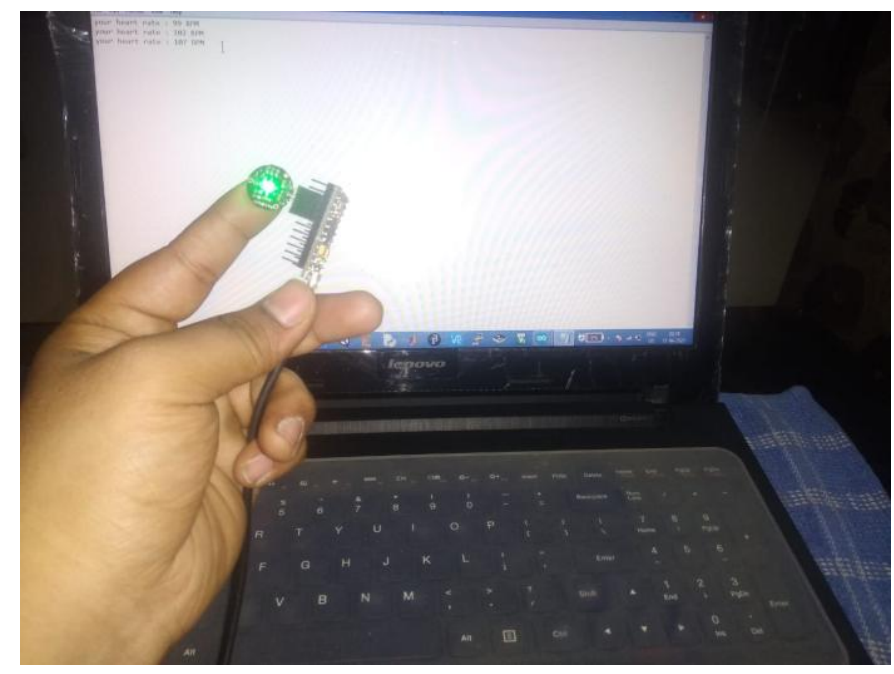

\section{CONCLUSION}

The device is working fine with mobile and PC. It is highly compact and power efficient and also a low cost device which is able to measure the pulse rate with good accuracy and working fine with different apps and with different devices. This device is highly portable and can be easily used by anyone no specific technical knowledge is required to operate the device. The device is very simple to operate. It is useful for everyone and can be carried easily. It does not require any kind of external battery.

\section{REFERENCES}

[1] "Kuo-Kai Shyu, Luan-Jiau Chiu, Po-Lei Lee, Member, IEEE, Tzu-Han and Shun-Han Yang”, Respiratory and pulse Rates using UWB radio detection and ranging sensing element information exploitation FVPIEF based mostly Two-Layer EEMD, IEEE 2018.

[2] Naoki Hagiyama , Akihisa Mito , Harutoyo Hirano, Zu Soh , Etsunori Fujita, Yumi Ogura , Ryuichi Uchikawa , Shigehiko Kaneko, "Unconstrained Monitoring of Biological Signals Using an Aortic Pulse Wave Sensor", IEEE 2018.

[3] Embedded Lab. Introducing Easy Pulse: A DIY photoplethysmographic sensor for measuring heart rate. http://embedded-lab.com/blog/?p=5508, 2012.

[4] Sankar Kumar S, Gayathri N, Nivedhitha D, Priyanka A S "A Cost effective Arduino Module for Bedridden patient's Respiratory Monitor and Control" International Journal of advanced research trends in engineering and technology (IJARTET) VOL. II, SPECIAL ISSUE XXI, MARCH 2015. 
ISSN (online): 2581-3048

Volume 5, Issue 4, pp 83-86, April-2021 https://doi.org/10.47001/IRJIET/2021.504013

[5] Ch.Sandeep Kumar Subudhi, "Intelligent Wireless Patient Monitoring and Tracking System (Using Sensor Network and Wireless Communication)", 2014.

[6] Bhagya Lakshmi.J.M1 Hariharan.R2 Udaya Sri.C3 Nandhini Devi.P4 Sowmiya.N "Heart Beat Detector using Infrared Pulse Sensor" IJSRD - International Journal for Scientific Research \& Development $\mid$ Vol. 3, Issue 09, 2015.

[7] Nazmus Saquib, Md. Tarikul Islam Papon, Ishtiyaque Ahmad, and Ashikur Rahman "Measurement of Heart Rate Using Photoplethysmography".

[8] Mehmet, T. IoT Based Wearable Smart Health Monitoring System. Celal Bayar Univ. J. Sci. 2018, 14, 343-350.

[9] Chao, L.; Xiangpei, H.; Lili, Z. The IoT-Based Heart Disease Monitoring System for Pervasive Healthcare Service. In Proceedings of the International Conference on Knowledge Based and Intelligent
Information and Engineering Systems, KES2017, Marseille, France, 6-8 September 2017.

[10] Arnob, S.; Akash, M.; Nilay, S.; Abhishek, K.K.; Binanda, K.M.; Souvik, C. An IOT based Portable Health Monitoring Kit. Int. J. Res. Appl. Sci. Eng. Tech. (IJRASET) 2018, 6, 701-708.

[11] Lee, W.K.; Yoon, H.; Han, C.; Joo, K.M.; Park, K.S. Physiological Signal Monitoring Bed for Infants Based on Load-Cell Sensors. Sensors 2016, 16, 409; doi:10.3390/s16030409.

[12] Zompanti, A.; Sabatini, A.; Santonico, M.; Grasso, S.; Gianfelici, A.; Donatucci, B.; Di Castro, A.; Pennazza, G. A Sensor Platform for Athletes' Training Supervision: A Proof of Concept Study. Sensors 2019, 19, 3948, doi:10.3390/s19183948.

[13] Kumar, A.; Balamurugan, R.; Deepak, K.C.; Sathish, K. Heartbeat sensing and Heart Attack detection using the internet of things (IoT). Int. J. Eng. Sci. Comput. (IJESC) 2017, 7, 6662-6666.

\section{Citation of this Article:}

Pranmya Ratnaparkhi, “USB Based Battery Independent Device for Pulse Measurement” Published in International Research Journal of Innovations in Engineering and Technology - IRJIET, Volume 5, Issue 4, pp 83-86, April 2021. Article DOI https://doi.org/10.47001/IRJIET/2021.504013 